\title{
Feature-Preserving Morphable Model for Automatic Font Generation
}

\author{
Rapee Suveeranont* \\ The University of Tokyo
}

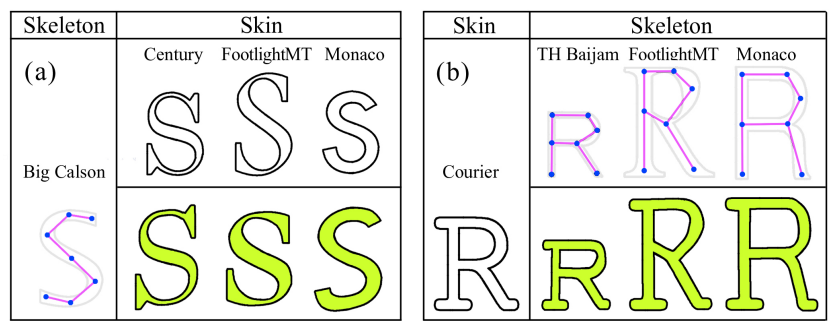

Figure 1: Synthesizing a new font (in green) by combining features (skin and skeleton) of template fonts

\section{Introduction}

Designing a digital font requires not only artistic but also technical skills. However, it is time-consuming and requires professional skills to create a nice font with consistency looks and plausible details. We present a font model that blends structures and outlines of template fonts individually, yet maintains the original style. We also present an algorithm to automatically generate font by blending the template fonts. It takes the desired outline of one character as an input and infer blending weights.

\section{Feature-Preserving Morphable Model}

Our font model has two main benefits. First, style and structure of a font are two independent components. They can be morphed and edited on their own (Figure 1). This contributes to larger font space that can be created from interpolation. Second, features are well-preserved and not deformed to a different structure.

Previous font morphing techniques use simple linear interpolation of an outline [Xu et al. 2005]. Instead, we morph by interpolating both structural and detailed parts independently. We represent them as an undirected graph skeleton and 2D coordinate parameter skin, respectively (Figure $2 a)$. We prohibit deformation (e.g. linear transformation) of features by simply fixing them to skeleton nodes. Non-feature areas are rotated and scaled in accordance with skeleton edges. Unfortunately, contour will get broken as seen in Figure $2 \mathrm{~b}$. We solve this problem by stitching skin between the holes. To be faithful to the original style, we treat skin in this area as a Laplacian contour, in the same manner as Laplacian surface [Sorkine et al. 2004]. We then solve the matrix equation of $L V=Q$ as a linear least square problem with smoothness constraints. As a result, the reconstructed contour will maintain curvature, smoothness and connectivity.

\section{Automatic Font Generation}

Given a database of sample fonts and one character outline of the desired new font as an input, we compute the blending weights to approximate the character. Provided by our model, we can fit the skeleton and skin separately. The fittest skeleton will limit search

\footnotetext{
*e-mail:iiieyes@gmail.com

†e-mail:takeo@acm.org
}

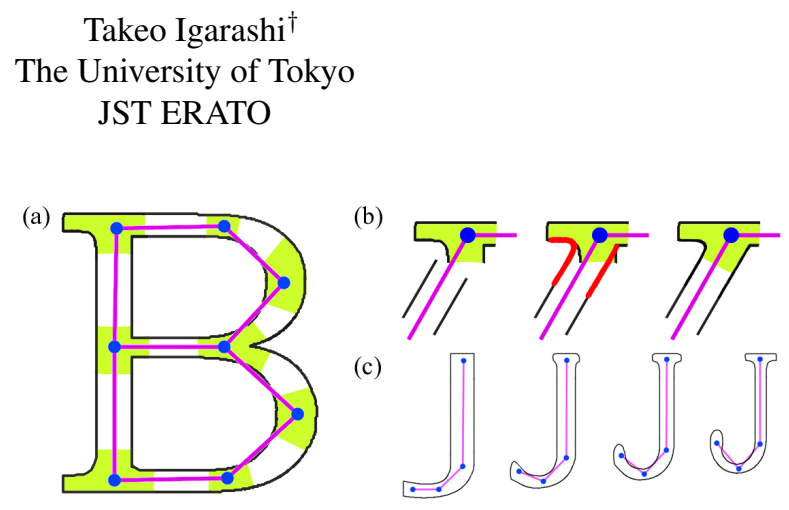

Figure 2: Feature-preserving morphable model composes of skeleton and skin with feature areas highlighted. (b) Broken contour and repair by Laplacian stitch. (c) Morphing skeleton and skin together.

scope and better approximate the final skin. This is a minimization problem and we solve by gradient descent method. With the assumption that blending contribution is consistent through out one font, we directly apply the optimized weights to the remaining characters. Our system provides user semi-automatic creation of a skeleton and a correspondence outline.

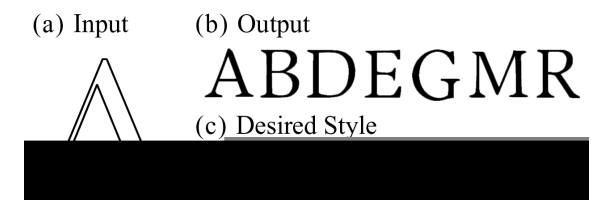

Figure 3: A character 'A' of Baskerville font, which was not included in our database, was used to test our algorithm. The output and desired style samples are presented for comparison.

\section{Results and Future Work}

Feature-preserving technique works well as long as the underlying structure is reasonable. There are 20 sample fonts in our testing database. Fonts in roman or san-serif styles gave promising results (Figure 3). On the other hand, user designed free-hand style were hardly captured very well. This is due to the fact that our database is too small. In the future, we want to improve our matching algorithm using more advanced techniques in order to generate fonts with wider range of styles. We plan to extend our algorithm to support multiple input characters and interactive editing interface. Finally, we would like to make the skeleton and correspondence outline creation an automatic process, which will ultimately leave user to focus only on the design.

\section{References}

Sorkine, O., Cohen-Or, D., Lipman, Y., Alexa, M., RÖSSL, C., AND SEIDEL, H.-P. 2004. Laplacian surface editing. In SGP '04: Proceedings of the 2004 Eurographics/ACM SIGGRAPH symposium on Geometry processing, ACM, New York, NY, USA, 175-184.

Xu, S., Lau, F. C. M., Cheung, W. K., And Pan, Y. 2005. Automatic generation of artistic chinese calligraphy. IEEE Intelligent Systems 20, 3, 32-39. 